\title{
NUCLEAR ACTIVITY IN INTERACTING GALAXIES
}

\author{
TAPAN K. CHATTERJEE \\ Facultad de Ciencias, Fisico-Matematicas, Universidad A. de Puebla, (A.P. 1916), Puebla, \\ Mexico
}

Dynamical studies of galactic collisions, conducted previously (Chatterjee, 1992, $1993 \mathrm{a}, \mathrm{b})$, indicated that most of the mergers take place in two to three shrinking orbital periods. We extend this line of research work to study induced nuclear activity. We study the binary evolution of a spiral galaxy perturbed by a compact elliptical galaxy of comparable mass and find that each time the perturber penetrates the disk of the spiral, the disk is subjected to an appropriate perturbation, causing inflow of gas towards its nucleus due to loss of angular momentum; there it could activate an inert black hole (consistent with previous studies; e.g. Naguchi, 1988). However a new feature that we find is that repeated episodes of disk penetration by the perturber occur in gradually shorter timescales, causing an overlap of the activity timescale and the dynamical timescale. In fact if the elliptical is very compact and the spiral has a massive bulge, subsequent dynamical timescales reduce by more than an order of magnitude. This periodic increase in the activity of the nucleus is of a secular nature (in contrast to a reactivation process), and could lead to the evolution of the spiral along the following lines, Starburst $\rightarrow$ Seyfert $2 \rightarrow$ Seyfert 1, as interpenetrations follow.

\section{References}

Chatterjee, T.K., 1992: Astrophys. Space Sci. 196, 283

Chatterjee, T.K., 1993a: Astrophys. Space Sci. 199, 35

Chatterjee, T.K., 1993b: Astrophys. Space Sci. 199, 189

Naguchi, M., 1988: Astron. Astrophys. 203, 259

T. J.-L. Courvoisier and A. Blecha: Multi-Wavelength Continuum Emission of AGN, 499.

(C) 1994 IAU. Printed in the Netherlands. 